\title{
Laser power loss through polystyrene plates for cell culture
}

\author{
Carla Andreotti Damante • Márcia Martins Marques
}

Received: 6 December 2012 / Accepted: 10 January 2013 /Published online: 19 January 2013

(C) Springer-Verlag London 2013

\section{Dear Editor,}

In 2009, we published a paper about in vitro release of fibroblast growth factors by human gingival fibroblasts [1]. The methodology for laser irradiation in cell cultures is to apply laser on the bottom of the 96-well plate avoiding the light transmission through the growth medium. Ideally the light would be delivered directly to the cell monolayer. The cell growth plates are made of polystyrene with a 1-mm width on the bottom. In that study, we used TPP plates (Trasadingen, Switzerland), and according to manufacturers, it has excellent optical characteristics, suitable for precise optical measurements, mainly on bottom reading. Challenged by this information, we investigated the interference of this polystyrene layer on low-level laser transmission. We cut a disk from the 96-well plate and introduced in a power meter (Laser Check power meter, Coherent, Inc., Santa Clara, CA, USA) to verify the output power of the laser equipment (MMOptics, São Carlos, Brazil). These measurements were compared to others made without the disk and we found interesting results. For visible red laser set in $40 \mathrm{~mW}$, the measurements were 44.1 and $38.8 \mathrm{~mW}$, without and with the disk, respectively. It showed a reduction of power of $12 \%$. For infrared laser set in $40 \mathrm{~mW}$, the measurements were 40.0 and $35.3 \mathrm{~mW}$, without and with the disk, respectively. This also revealed a $12 \%$ loss of power. These differences were statistically significant $(p<0.05)$. We consider that this information is fundamental to studies with laser and culture cells. The percentage of power loss must be considered and added to the final power settings in the device.

\section{References}

1. Damante CA, De Micheli G, Miyagi SPH, Feist IS, Marques MM (2009) Effect of laser phototherapy on the release of fibroblast growth factors by human gingival fibroblasts. Lasers Med Sci 24(6):885-891
C. A. Damante $(\square)$

Faculdade de Odontologia de Bauru, Bauru School of Dentistry,

Al. Octavio Pinheiro Brisolla 9-75,

17012-901, Bauru, Sao Paulo, Brazil

e-mail: cdamante@rocketmail.com

M. M. Marques

Department of Endodontics, University of São Paulo, Sao Paulo, Brazil 